\title{
Síndrome da fragilidade no idoso: importância da fisioterapia
}

Frailty syndrome in elderly patients: the importance of physiotherapy

Camila Macedo, Juliana Maria Gazzola, Myrian Najas

\section{Resumo}

A fragilidade é conceituada como uma síndrome clínica, que pode ser identificada por perda de peso involuntária, exaustão, fraqueza, diminuição da velocidade da marcha e do equilíbrio e diminuição da atividade física. Cada uma dessas manifestações clínicas é preditora de uma série de reações adversas (quedas, hospitalização, institucionalização, declínio funcional e morte). 0 objetivo desta revisão de literatura foi expor os elementos sobre a Síndrome da Fragilidade dos Idosos, em uma revisão narrativa da literatura, dando um panorama geral sobre epidemiologia, tendências atuais, ciclo da fragilidade, sarcopenia, alterações imunológicas, alterações neuroendócrinas, diagnósticos clínico, laboratorial e diferencial e as possibilidades de intervenções fisioterapêuticas. Para esses fins, realizou-se revisão bibliográfica da literatura nacional e internacional sobre o tema. Com o conhecimento de estudos envolvendo idosos frágeis, é possível planejar as estratégias voltadas para a identificação da parcela da população que mais se beneficiaria de ações para postergar, atenuar e, se possível, reverter tais desfechos.

Palavras-chave: Idoso; fragilidade; envelhecimento; sarcopenia; exercício físico.

\section{Abstract}

The frailty syndrome is a clinical syndrome that may be identified by involuntary loss of weight, exhaustion, weakness, decrease of walking rate and balance, and decrease of physical activity. Each one of these clinical manifestations predicts a series of adverse reactions (falls, hospitalization, institutionalization, functional decline, and death). The aim of this literature review was to expose the elements on Frailty Syndrome in the elderly in a narrative literature review, providing a general panorama upon epidemiology, actual trends, frailty cycle, sarcopenia, immunological disorders, neuroendocrinal disorders, clinical, laboratorial and differential diagnosis, and the possibilities of physiotherapeutic intervention. Therefore, a national and international literature review on the subject was performed. By knowing studies involving fragile elderly patients, it is possible to plan strategies towards the identification of parcel of the population that would benefit the most from actions to delay, to attenuate and, if possible, to revert such manifestations.

Keywords: Elderly; frailty, aging; sarcopenia; physical exercise.

Recebido: 04/06/2008

Revisado: $16 / 10 / 2008$

Aprovado: 27/10/2008 


\section{Introdução}

O termo fragilidade tem sido utilizado na prática para designar, dentre a população de idosos, aqueles que apresentam características clínicas atribuídas ao envelhecimento, associado à existência de comorbidades, como por exemplo, diminuição da massa e da força muscular, exaustão, alteração da marcha e do equilíbrio, anorexia, perda de peso progressiva. Todos esses fatores levam a um maior risco de eventos adversos como quedas, incontinência urinária, hospitalização e morte ${ }^{1}$. A fragilidade está associada à idade, embora não seja resultante exclusivamente do processo de envelhecimento, já que a maioria dos idosos não se torna frágil obrigatoriamente. Ela está relacionada com a presença de comorbidades, pois as doenças crônicas que surgem nas fases mais avançadas da vida tendem a ser menos letais e a se acumularem durante o processo de envelhecimento.

Este estudo teve como objetivo fazer uma revisão sobre os elementos da Síndrome da Fragilidade dos Idosos, dando um panorama geral sobre epidemiologia, tendências atuais, ciclo da fragilidade, sarcopenia, alterações imunológicas, alterações neuroendócrinas, diagnósticos clínico, laboratorial e diferencial e as possibilidades de intervenções fisioterapêuticas. Para tanto, realizou-se revisão bibliográfica narrativa da literatura nacional e internacional sobre o tema, consultando as bases de dados MEDLINE, PubMed e LILACS e utilizando-se das palavraschave: idoso, fragilidade, envelhecimento, sarcopenia e exercício físico. As publicações utilizadas referem-se ao período dos últimos dez anos. O estudo também apresenta referências de livros textos e dissertações de mestrado.

\section{Revisão}

\section{Fragilidade como conceito}

Não há consenso definido quanto ao termo fragilidade, nem como poderia ser identificada ou mesmo avaliada. As primeiras definições que utilizaram critérios para indicação de fragilidade consideraram idosos frágeis, indivíduos com mais de 65 anos de idade, dependentes para atividades de vida diária (AVD) e geralmente institucionalizados ${ }^{2}$.

Mais recentemente, para ser considerado frágil, o idoso deve atender a um critério, dentre vários. Deve-se incluir a doença crônica incapacitante, o estado de confusão mental, a depressão, as quedas, a incontinência urinária, a desnutrição, as úlceras por pressão e os problemas socioeconômicos ${ }^{3}$. Desta forma, a fragilidade pode ser observada quando o idoso preenche no mínimo quatro das seguintes características: idade igual ou superior a 80 anos, depressão, instabilidade de equilíbrio e marcha, diminuição da força de preensão palmar, uso de sedativos, diminuição da força nas articulações dos ombros e joelhos, déficits nos membros inferiores (MMII) e déficit visual ${ }^{4}$
A fragilidade é uma condição instável relacionada ao declínio funcional. A interação do indivíduo com o ambiente, na qual a ocorrência de um evento, considerado de pequeno impacto para alguns idosos, pode causar limitação no desempenho das AVD e resultar ou não na perda da autonomia ${ }^{5}$.

O déficit físico não está relacionado com fragilidade, pois a condição pode ser definida como resultado de interação entre recursos e perdas de capacidades que tornam o indivíduo mais vulnerável aos desafios ambientais ${ }^{6}$.

Fragilidade é considerada, por muitos autores, estado clínico de vulnerabilidade aos fatores estressantes que resultam no declínio das reservas fisiológicas, com subseqüente diminuição da eficiência da homeostase. Nos indivíduos, a fragilidade indica uma condição prevalente em mulheres, caracterizada por alto risco para conseqüências adversas, quedas, incapacidade, hospitalização, institucionalização e morte $\mathrm{e}^{1,7}$.

Uma expressão complexa e cumulativa de respostas homeostáticas alteradas aos múltiplos estressores, resultando em desequilíbrio metabólico, pode ser resultante da fragilidade ${ }^{8}$. A característica essencial de fragilidade é a noção do risco decorrente da instabilidade?. O idoso torna-se frágil quando um organismo perde a complexidade de manutenção de sua dinâmica e apresenta respostas de não adaptação aos estresses ${ }^{10}$.

Em geral, os idosos percebidos como frágeis são aqueles que apresentam riscos mais elevados para desfechos clínicos adversos, tais como: dependência, institucionalização, quedas, piora do quadro de doenças crônicas, doenças agudas, hospitalização, lenta ou ausente recuperação de um quadro clínico e morte ${ }^{11,7}$.

O conceito de fragilidade não é novo, mas é recente a sistematização de informações que possibilitam a observação de que um idoso está frágil e consequentemente, vulnerável aos efeitos adversos de estresses considerados de menor impacto ${ }^{12}$.

Fragilidade pode ser considerada quando, sob condições de estresse, um indivíduo tem reduzida sua habilidade para executar importantes práticas de atividades instrumentais de vida diária (AIVD) ${ }^{13}$. Isso representa uma forma de pré-incapacidade e, como tal, precisa ser distinto da redução de capacidade funcional.

O conhecimento sobre os eventos que resultam nesta condição é limitado e alguns pesquisadores vêm buscando de forma intensa a identificação da etiologia e as características dessa síndrome $\mathrm{m}^{7-11,13}$.

\section{Epidemiologia}

Existem ainda poucos dados disponíveis sobre a incidência e prevalência da Síndrome da Fragilidade em idosos, principalmente pela falta de consenso de uma definição que possa ser utilizada como screening em diferentes populações. A partir de dados do Cardiovascular Health Study, estimou-se que em uma população com idade superior a 65 anos, 6,3\% dos idosos tinham o fenótipo de fragilidade. Entre os 
frágeis, $68,5 \%$ eram mulheres. Houve evidência do aumento de prevalência de fragilidade com a idade ${ }^{1}$.

Recentemente em outro estudo epidemiológico com mulheres de 65 a 79 anos, em que os critérios de fragilidade foram bem observados, evidenciou-se a relação entre fragilidade e doença cardíaca isquêmica, acidente vascular encefálico (AVE), doença pulmonar obstrutiva crônica (DPOC), diabetes mellitus (DM), hipertensão arterial e a osteoartrite. A prevalência de incapacidades físicas foi maior entre os frágeis, quando comparado aos não-frágeis. $\mathrm{O}$ aumento no risco de fragilidade associou-se positivamente com baixo nível socioeconômico, antecedente de doença isquêmica cardíaca, DM, fratura de quadril, DPOC, osteoartrite, AVE e depressão ${ }^{14}$.

\section{Tendências atuais no estudo da fragilidade}

Dois grupos de pesquisa têm se destacado na busca de consenso sobre a definição de fragilidade: nos Estados Unidos, na Johns Hopkins University, e no Canadá, o Canadian Iniciative on Frailty and Aging (CIF-A).

A pesquisa Canadian Iniciative on Frailty and Aging (CIF-A) é uma colaboração desenvolvida entre Canadá, União Européia, Israel e Japão, com o objetivo de ampliar o conhecimento sobre fragilidade em idosos, por meio de estudo minucioso das causas e da trajetória do fenômeno, com ênfase em sua prevenção e tratamento. Segundo Berg$\operatorname{man}^{15}$, as pesquisas são realizadas por diferentes especialistas, subdivididas em 11 domínios: 1) história, conceitos e definições; 2) bases biológicas; 3) serviços de saúde; 4) bases sociais; 5) prevalência; 6) história natural e fatores de risco; 7) impacto da fragilidade; 8) identificação; 9) prevenção e conduta terapêutica; 10) ambiente e tecnologia; 11) política social e de saúde.

Embora ainda esteja em andamento, os membros da CIF-A desenvolveram um construto multidimensional enfatizando a etiologia complexa da fragilidade, relacionando-a com a história de vida de idosos e considerando que essa trajetória pode ser modificada por fatores biológicos, psicológicos e sociais, cujas interações resultam em recursos e/ou déficits individuais em determinado contexto ${ }^{16}$.

Iniciando-se em fase subclínica (condição pré-frágil) o processo pode evoluir, sem muita precisão temporal para as manifestações clínicas. O maior potencial para prevenção encontra-se no estágio que antecede essas manifestações. Após a instalação do fenômeno, a conduta terapêutica tem por objetivo, prevenir, adiar ou amenizar os efeitos adversos ${ }^{16}$.

Apoiando-se nesse modelo conceitual, Teixeira e Néri ${ }^{16}$ consideram que o conceito de fragilidade está sendo reconstruído como multifatorial, distanciando-se do dominante paradigma biomédico. Para os autores, indicadores objetivos para medir fragilidade física não podem ser reduzidos em variáveis mensuráveis. Por exemplo, a avaliação do desempenho nas atividades básicas de vida diária responsabilizaria excessivamente o idoso pelas conseqüências advindas da condição, difi- cultando a compreensão de que há um contexto sociocultural importante exercendo influência na saúde do indivíduo.

Já o grupo de pesquisadores da Johns Hopkins University nos Estados Unidos, propôs critérios objetivos mensuráveis para definir fragilidade em idosos a partir da hipótese de que esta representa uma síndrome que pode ser identificada a partir de um fenótipo ${ }^{1}$.

Para os pesquisadores, a fragilidade é síndrome clínica caracterizada pela diminuição de reserva energética e resistência reduzida aos estressores. Essa condição resulta de declínio cumulativo dos sistemas fisiológicos e causa vulnerabilidade às condições adversas, por haver dificuldade de manutenção da homeostase em situações de exposição às perturbações, tais como alterações de temperaturas ambientais e variações na condição de saúde ${ }^{1}$.

São três as principais mudanças relacionadas à idade, subjacentes às síndromes: 1) alterações neuromusculares (principalmente sarcopenia); 2) desregulação do sistema neuroendócrino; 3) disfunção do sistema imunológico.

É excluída do modelo o déficit cognitivo grave, uma vez que, conseqüências da demência surgirão como condições clínicas características da síndrome.

Esse grupo de pesquisadores construiu um fenótipo relacionado à fragilidade, incluindo cinco componentes com possibilidade de serem mensurados: 1) perda de peso não intencional: maior de $4,5 \mathrm{~kg}$ ou superior a $5 \%$ do peso corporal no último ano; 2) fadiga auto-referida; 3) diminuição da força de preensão palmar, medida com dinamômetro e ajustada para gênero e índice de massa corporal (IMC); 4) baixo nível de atividade física medida pelo dispêndio semanal de energia em kcal (com base no auto-relato das atividades e exercícios físicos realizados) e ajustado segundo o gênero; 5) diminuição da velocidade de marcha em segundos: distância de $4,5 \mathrm{~m}$ ajustada para gênero e altura.

Desta forma o fenótipo seria resultado de um ciclo, cujo início ainda não está claramente especificado. O ciclo é representado por espiral com potencial decrescente de reserva de energia de múltiplos sistemas e explica, hipoteticamente, as condições de fadiga, perda de peso, e alterações da velocidade da marcha, justificando o alto risco para as conseqüências adversas da síndrome. O indivíduo que apresenta três ou mais componentes do fenótipo enquadra-se em idosos frágeis e aqueles com um ou dois componentes seriam indicativos de alto risco de desenvolver a síndrome.

O ciclo da fragilidade (Figura 1) mostra os principais componentes desta síndrome: desnutrição crônica, sarcopenia, declínio da massa e da força muscular e tolerância ao exercício; além do declínio da atividade física e do gasto energético total ${ }^{1,7}$.

Este ciclo descreve um processo de perda energética que inclui perda de massa e força muscular, diminuição da taxa metabólica, declínio do gasto energético e da mobilidade. Nesse modelo estão múltiplos pontos potenciais de entrada no ciclo, que incluem condições fisiológicas subjacentes que são centrais à síndrome, como estados 


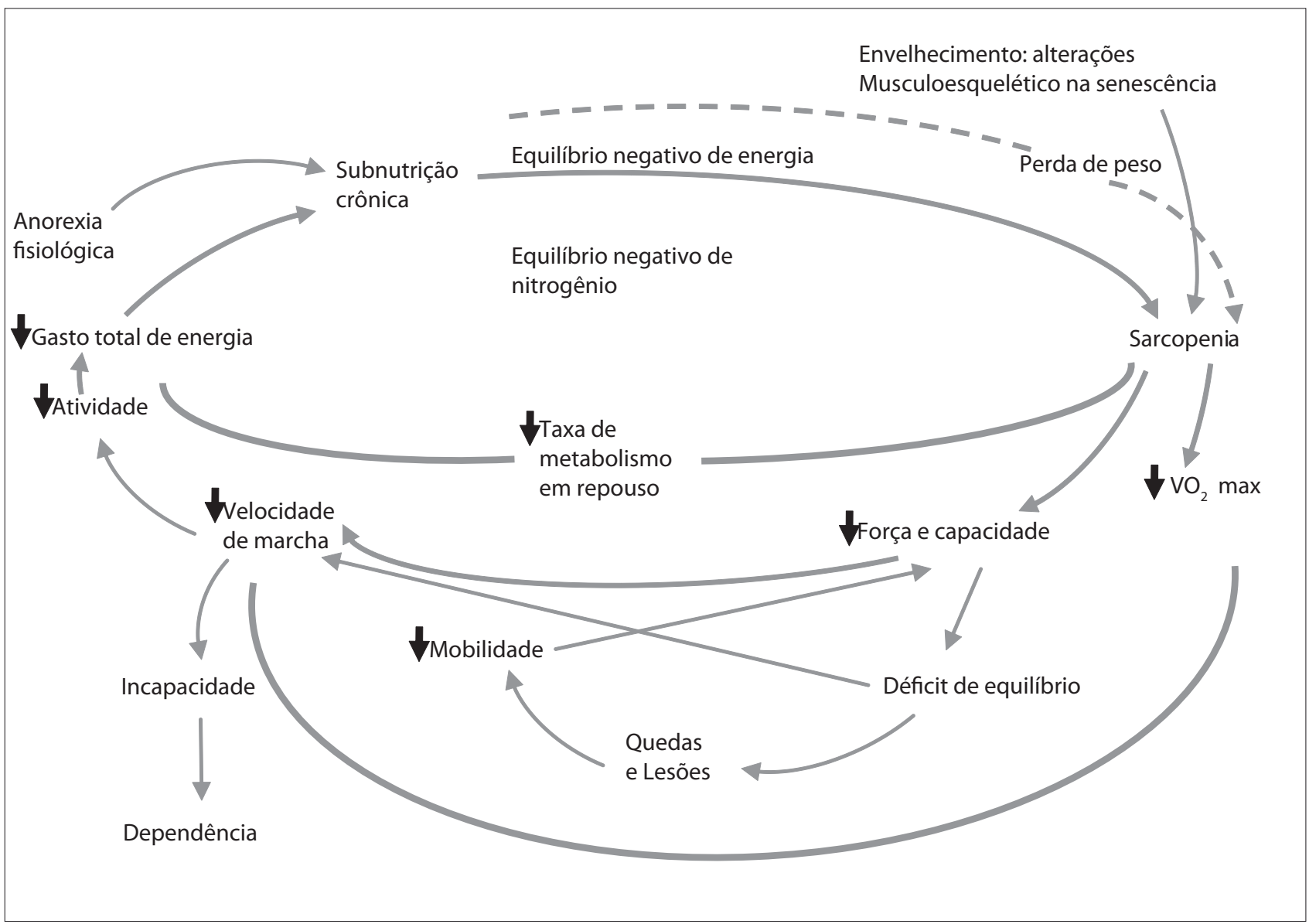

Figura 1: Interrelações no ciclo da fragilidade (Adaptado de Fried \& Walston ${ }^{12}$ )

patológicos, imobilidade, depressão e medicações, que podem desencadear ou acelerar este processo. Nele também podem ser encontradas muitas manifestações clínicas da fragilidade. Contudo, como é um modelo teórico, não é capaz de explicar a existência de todas elas ${ }^{1,7}$.

Os principais componentes na retroalimentação negativa deste ciclo são: a subnutrição crônica, a sarcopenia, o declínio da força física e da tolerância ao exercício, e o declínio no gasto total de energia. ${ }^{18}$

A fragilidade, as comorbidades e as incapacidades são condições clínicas distintas, embora possam ocorrer simultaneamente.

\section{Principais componentes da Síndrome da Fragilidade}

\section{Sarcopenia}

A sarcopenia está associada à fragilidade e tem com conseqüências: diminuição da força muscular, baixa tolerância ao exercício e redução da velocidade da marcha ${ }^{18}$. Walston et al. ${ }^{19}$ consideram que a sarcopenia consiste em múltiplos mecanismos no nível molecular-celular, sistêmico e orgânico: danos oxidativos do DNA mitocondrial, senescência celular, desregulação hormonal e desnutrição.

A sarcopenia é uma das variáveis utilizadas para definição da Síndrome de Fragilidade, sendo altamente prevalente em idosos e conferindo maior risco para quedas, fraturas, incapacidade, dependência, hospitalização recorrente e mortalidade. É definida como perda de massa e força muscular. Tem sido associada à atrofia das fibras musculares rápidas (tipo II a) e à substituição por tecido adiposo e fibrótico com diminuição da síntese protéica, ocasionando redução da força e eficiência muscular. Observa-se ainda uma alteração na qualidade das fibras musculares, na efetividade neural, no controle fino do equilíbrio e na diminuição das aferências sensitivas e motoras ${ }^{20}$. Esta situação acomete frequentemente o idoso, sendo considerada a principal causa do aumento da prevalência da incapacidade.

A sarcopenia tem um grande impacto na capacidade funcional de um indivíduo. A diminuição da força muscular e da tolerância ao exercício leva à diminuição da capacidade das AVD e conseqüentemente ao aumento da dependência.

Os músculos formam uma importante reserva de proteínas corpóreas, que podem ser utilizadas em situações extremas para produção de anticorpos e energia, além de ser o local de metabolização de uma série de hormônios ${ }^{20}$.

Estudos mostram que níveis elevados de interleucina (IL6) foram preditores do declínio funcional acelerado em idosos, por meio da perda da massa e principalmente da força muscular. A perda de massa 
muscular pode ser acompanhada de ganho progressivo de massa gorda e aumento da resistência periférica à insulina em idosos ${ }^{21}$.

\section{Alterações imunológicas}

O envelhecimento tem sido associado a um aumento crônico dos níveis circulantes de marcadores inflamatórios, incluindo citoquinas pró-inflamatórias e antiinflamatórias, antagonistas de citoquinas e proteínas de fase aguda ${ }^{21}$.

A grande variação de fatores ambientais, incluindo tabagismo, infecções, obesidade, fatores genéticos, declínio funcional, fragilidade e diminuição de hormônios sexuais pode contribuir para atividade inflamatória sistêmica em idosos ${ }^{21}$.

Uma ativação imune generalizada relacionada ao envelhecimento pode contribuir para o desenvolvimento da Síndrome de Fragilidade por meio de influências patológicas em outros órgãos e sistemas ${ }^{22}$.

Várias evidências confirmam o papel direto do TNF- $\alpha$ (fator de necrose tumoral) na indução de um estado catabólico que causa fragilidade, além de sua associação na patogênese da aterosclerose, DM tipo II e Doença de Alzheimer em idosos ${ }^{23}$.

A ativação inflamatória generalizada ocorreria como conseqüência da falha de mecanismos regulatórios que permitem que células imunes ativadas continuem a produzir agentes catabólicos, mesmo depois de cessado o estímulo. A exposição em níveis elevados dessas substâncias por um longo período contribui para a fragilidade, pela sua ação catabólica ${ }^{21}$.

Idosos saudáveis com altos níveis circulantes de TNF- $\alpha$, IL-6 e proteína C-reativa predizem morbidade e mortalidade, independente de comorbidade. Os níveis circulantes de TNF- $\alpha$ representam o melhor indicador de mortalidade em idosos frágeis, enquanto que níveis de IL-6 são um bom marcador para idosos saudáveis ${ }^{24}$.

Roubenoff et al. ${ }^{25}$, em um estudo de análise longitudinal, observaram que baixos níveis de IGF-1 (fator 1 do hormônio de crescimento) e elevados de IL-6 foram associados à perda mais rápida do índice de massa magra. Um subestudo do Women's Health and Aging Study mostrou que IL-6 associou-se com a mortalidade na presença de doença cardiovascular. Já nos idosos extremamente saudáveis, a produção de IL-6 não diferiu dos indivíduos jovens. Estes resultados confirmam a falta de clareza na identificação das causas da elevação dos níveis de IL-6.

Em outro estudo com 3075 indivíduos, com idades entre 70-79 anos, os autores demonstraram a associação dos altos níveis de IL-6 e TNF- $\alpha$ à diminuição de massa muscular e de força muscular, tanto em homens como em mulheres ${ }^{26}$.

Recentes estudos sugerem que a IL-10 é a citoquina chave que pode suprimir a imunidade mediada por células. É responsável pela maturação das células dendríticas e tem sido encontrada em níveis elevados em idosos saudáveis ${ }^{23}$

\section{Alterações neuroendócrinas}

Com o envelhecimento, ocorre um declínio na secreção dos hormônios testosterona, estrogênio, hormônio luteinizante e do DHEA (dehidroepiandrosterona), associado ao aumento dos níveis de cortisol, e estas alterações têm sido indicadas como fatores particularmente envolvidos no desenvolvimento da fragilidade ${ }^{27}$.

O sistema neuroendócrino é altamente complexo e integrado. É capaz de avaliar informações ambientais e sensoriais mantendo um balanço homeostático pelas vias nervosa e endócrina aos sistemas de órgãos-alvo. Muitas modificações evidenciadas no processo de envelhecimento estão relacionadas com a perda de mecanismos coordenados e na regulação fina destes sistemas ${ }^{22}$. A mais importante resposta neuroendócrina ao estresse é a ativação do sistema nervoso simpático, com elevação dos níveis plasmáticos de glicocorticóides. Esse fenômeno pode causar diversos problemas como: danos físicos (infecção, inflamação, hipotermia), psicológicos (ansiedade, depressão, isolamento social) e dor. Os sintomas que se observa são: taquicardia, dilatação brônquica e aumento da força muscular, todos relacionados à estimulação dos $\beta$-receptores adrenérgicos. Quando o sistema está perfeitamente regulado, a função dessas respostas é minimizar o impacto das situações de risco. Entretanto, quando esses efeitos passam a ser crônicos, tornam-se deletérios. No envelhecimento é bem conhecido um estímulo crônico do sistema nervoso simpático, em que os níveis de epinefrina e norepinefrina estão constantemente elevados. A desregulação da resposta é evidente nos níveis mais elevados de cortisol em idosos, ocasionando uma desregulação da resposta ao estresse.

Outros hormônios associados à Síndrome da Fragilidade são: GH (hormônio do crescimento), secretado a partir do estímulo pulsátil do GHRH, produzido e liberado de maneira pulsátil na hipófise, e sua maior molécula mensageira: o IGF-1 (fator de crescimento semelhante à insulina isoforma 1). Tais hormônios têm um papel fundamental no desenvolvimento e na manutenção da massa muscular. Com o envelhecimento, ocorre diminuição da secreção pulsátil de GH e IGF-1 e a disfunção deste eixo pode mediar a alteração na massa e força muscular observada em indivíduos frágeis ${ }^{23}$.

\section{Suplementos nutricionais}

O estado nutricional é freqüentemente comprometido nos idosos frágeis. Inúmeros são os fatores que podem ser responsáveis por esse quadro, como: o efeito colateral de medicamentos, o comprometimento visual, a diminuição do paladar, a diminuição do olfato, os problemas dentários, a diminuição da salivação, a depressão, o isolamento social, o alcoolismo, a anorexia, a incapacidade física, as dificuldades financeiras ou sociais e as doenças crônicas avançadas ${ }^{20}$.

Um dos suplementos nutricionais mais discutidos para o uso na Síndrome da Fragilidade é a creatinina. Isso porque ela tem um possível efeito de elevar o depósito de fosfocreatina no músculo, além de poder aumentar níveis de adenosina tri-fosfato (ATP) e fosfocreatina durante o exercício. Os efeitos benéficos potenciais desse tipo de intervenção, parecem ocorrer especialmente quando a suplementação nutricional está associada ao exercício físico. A literatura não dispõe de estudos com ênfase na função muscular propriamente dita ${ }^{28}$. 
O picolinato de cromo, outra substância utilizada anedoticamente para definição da função muscular, mostrou-se ineficaz em idosos, tanto para o ganho de massa muscular quanto para redução da massa adiposa ${ }^{29}$.

\section{Diagnóstico laboratorial}

Até o momento não existem exames laboratoriais específicos para o diagnóstico de fragilidade. Marcadores laboratoriais estariam relacionados com a fisiopatologia da síndrome, associados ao declínio nutricional, alterações neuroendócrinas, alterações músculo-esqueléticas, hematológicas e imunológicas. Os marcadores laboratoriais que podem estar presentes na Síndrome da Fragilidade são: albumina; pré-albumina; transferrina, ceruplasmina, folato, zinco e vitaminas; colesterol total e frações; triglicérides; IGF-1-GH; insulina de jejum e após teste de tolerância à glicose; IL-6; proteína C-reativa; dímero D; fibrinogênio; marcadores genéticos: single nucleotide polymorphism (SNP) e mutação de DNA mitocondrial ${ }^{14}$.

\section{Critérios clínicos}

Considera-se frágil aquele indivíduo que apresentar três ou mais características discutidas anteriormente, e, pré-frágil àquele com uma ou duas dessas características ${ }^{1}$. As manifestações clínicas mais freqüentes na Síndrome da Fragilidade são: perda de peso não intencional, fraqueza muscular, fadiga, percepção de exaustão, anorexia, inatividade física e alterações de marcha e equilíbrio.

Individualmente, cada uma das manifestações clínicas é preditora de uma série de eventos adversos, relacionados com a Síndrome da Fragilidade, como o declínio funcional, institucionalização, quedas e morte ${ }^{1,7,14}$.

O baixo desempenho em alguns testes, como testes funcionais de performance, velocidade da marcha, avaliação do equilíbrio estático, medida do tempo para se levantar cinco vezes de uma cadeira e a força de preensão palmar, mostrou um risco independente de declínio funcional futuro, mesmo entre indivíduos funcionalmente independentes ${ }^{1}$.

As medidas de função física parecem integrar os efeitos das múltiplas facetas da saúde e do envelhecimento, como condicionamento físico, impacto de diversas doenças e estado emocional ${ }^{1}$. Como um sinal vital, ela seria um indicador que poderia refletir os efeitos desses diversos processos fisiológicos e fisiopatológicos na funcionalidade do indivíduo.

\section{Diagnóstico diferencial}

O diagnóstico diferencial é feito descartando-se doenças consumptivas, terminais e, na possibilidade da identificação de componentes suficientes que preencham a Síndrome da Fragilidade. Fazem parte do diagnóstico diferencial: as doenças malignas; as psiquiátricas, especificamente a depressão; as gastrointestinais; as endócrinas, especialmente o hipertiroidismo; as cardiovasculares; o alcoolismo; as desordens nutricionais; as respiratórias; as neurológicas; as infecções crônicas; as doenças renais; aquelas do tecido conectivo; e o efeito colateral dos medicamentos ${ }^{1}$.

\section{Intervenções fisioterapêuticas}

Como não há consenso sobre a definição e a etiologia da fragilidade, os resultados das estratégias e dos tratamentos fisioterapêuticos foram alcançados sem a definição precisa da síndrome?.

As características individuais e o grau de fragilidade devem ser observados na definição dos planos de tratamento, sejam elas a curto ou longo prazo ${ }^{12}$.

A perda de massa muscular é acentuada nos idosos frágeis, mas estudos têm mostrado que os exercícios físicos são benéficos para os idosos nessa condição de saúde. O tratamento fisioterapêutico tem apresentado resultados significativos nessa população, levando ao aumentando da amplitude de movimento (ADM), melhor desempenho na realização das AVD, melhora na velocidade da marcha, melhora do equilíbrio, redução no número de quedas e bem-estar geral ${ }^{30}$.

É fundamental que durante o processo de envelhecimento, ocorra a maior concentração possível de massa muscular para que se possa retardar a perda inexorável decorrente dela mesma e assim promover menor impacto sobre a qualidade de vida dos idosos ${ }^{20}$. Desta forma, vale a pena ressaltar que a prevenção é a estratégia mais importante e eficiente para atingir esses objetivos.

\section{Treinamento de força muscular}

$\mathrm{O}$ treinamento do exercício físico resistido tem sido cada vez mais indicado para idosos, como uma maneira eficaz e segura de melhorar a força muscular e a capacidade funcional ${ }^{11,32}$. O aumento da capacidade do músculo de gerar força é explicado a partir do princípio de sobrecarga; o grupo muscular é submetido a um trabalho com cargas mais elevadas do que está acostumado a suportar, gerando aumento de tamanho e força ${ }^{32}$.

Estudos revelaram melhora significativa da força do quadríceps e de tempo de caminhada, em treino com carga versus nenhum treino ${ }^{33}$.

Indivíduos muito idosos (média de idade de 87 anos), institucionalizados, que realizaram treino de resistência associado à suplementação nutricional, por dez semanas, tiveram aumento de força muscular, bem como melhora objetiva da marcha, velocidade e atividade física espontânea. Os potenciais benéficos a longo prazo são: o menor número de quedas, aumento da mobilidade e independência ${ }^{34}$.

Pesquisas mostram aumento da síntese protéica muscular em resposta ao treino de resistência. Mudanças na inervação e padrão de ativação muscular também ocorrem com o treinamento, melhorando o desempenho motor ${ }^{35}$.

$\mathrm{O}$ treinamento de força deve ser realizado pelo menos duas vezes por semana, com um mínimo de 48 horas de repouso entre as sessões, para a recuperação da musculatura e prevenção do super treinamento. Recomenda-se realizar de oito a dez exercícios com oito a 12 repetições para cada conjunto. A seleção dos grupos a serem trabalhados deve ser direcio- 
nada aos grandes grupos musculares, sendo que a duração das sessões não deve ultrapassar a 60 minutos. Recomenda-se inspirar antes de levantar a carga e expirar durante a contração, evitando a manobra de valsalva ${ }^{36}$.

A prática de exercícios de resistência ainda é a intervenção mais efetiva para aumentar a massa e força muscular em idosos. É importante ressaltar que alguns idosos podem ter ingestão alimentar reduzida e necessidades protéicas aumentadas, dificultando a obtenção dos efeitos do treinamento de resistência se a nutrição não for adequada $a^{36,37}$.

$\mathrm{O}$ aumento das habilidades funcionais com treinamento de força somente tem sido verificado para indivíduos frágeis. As posições em velocidades em que os exercícios de força são realizados não são relevantes para a execução das AVD e AIVD, indo ao encontro do princípio de especificidade do treinamento ${ }^{38,39}$.

Em estudo de treinamento de força, com indivíduos idosos, para verificar as influências sobre os riscos de quedas, evidenciou-se que a intensidade do treinamento deve ser progressiva, com uma freqüência de três vezes por semana e intensidade de $50 \%$ de uma repetição máxima (RM), passando para $80 \%$ de uma RM nas duas últimas semanas. Neste estudo, verificou-se um aumento de 32 a $48 \%$ de força muscular $^{40}$. $\mathrm{O}$ treinamento de alta intensidade vem sendo indicado para promover ganhos de força máxima, no entanto, neste estudo os resultados demonstraram que, o treinamento de baixa a moderada intensidade mostrou-se tão eficaz quanto os de alta para esta finalidade. Quanto aos testes funcionais, os resultados foram estatisticamente significativos ( $\mathrm{p}$ $<0,05)$, indicando que o treinamento de força pode estar diretamente associado à diminuição do risco de quedas ${ }^{40}$.

\section{Equilíbrio e velocidade de marcha}

O treinamento da capacidade aeróbia tem se mostrado efetivo para diminuir a taxa de quedas e também para modificar os seus fatores de risco, em função dos ganhos de força muscular, mobilidade articular, aumento da velocidade da marcha e melhora na qualidade do equilíbrio ${ }^{39}$.

A perda da capacidade aeróbia dificulta a execução das AVD e AIVD (por exemplo, subir escadas, vestir-se, atravessar a rua), pois para realizá-las é necessário o condicionamento cardiovascular. Com base nisto, o treinamento da capacidade aeróbia seria uma estratégia de prevenção de quedas.
Alguns estudos têm mostrado aumento das habilidades funcionais quando o treinamento de força é combinado com outros tipos de treinamento; sua associação ao treinamento da capacidade aeróbia e do equilíbrio tem se mostrado mais eficiente para aumentar o controle do equilíbrio e a velocidade da marcha ${ }^{39,41}$.

Os exercícios para treino de equilíbrio devem ter duração de dez a 30 segundos com duas a três repetições para cada posição ou exercício, perfazendo um total de dez a 15 minutos. Os exercícios de equilíbrio podem ser estáticos e/ou dinâmicos, que envolvam alterações no input visual, mudanças na superfície de sustentação e redução da base de sustentação, e aumentem gradativamente o nível de dificuldade e complexidade das posições $^{37}$. Assim podem ser propostas para a terapia: aumentar o tempo em cada posição, utilizar espumas de densidades e materiais diferentes, posicionar a cabeça em diferentes angulações, usar diferentes conflitos visuais, variar a base de sustentação, entre outros recursos.

\section{Considerações finais}

As definições e os modelos de fragilidade evidenciam que a síndrome é complexa. A fragilidade é uma síndrome decorrente da interação de fatores biológicos, psicológicos, cognitivos e sociais, ao longo do curso da vida que poderia ser identificada precocemente para que intervenções e condutas também precoces fossem realizadas. Entretanto, apesar de não existir até o momento um tratamento específico para esta síndrome, a realização periódica de uma avaliação geriátrica global por uma equipe multidisciplinar é capaz de retardar o declínio funcional e prevenir a fragilidade, e assim diminuir o índice de institucionalização e hospitalização, alterando positivamente as taxas de morbimortalidade para esta parcela de população. Atualmente, a prática do treinamento do exercício físico resistido em membros inferiores é considerada como o mais eficaz método para preservar a mobilidade e, consequentemente, prevenir o declínio funcional em idosos. A fisioterapia tem importante papel na reabilitação dos pacientes com a Síndrome da Fragilidade, auxiliando tanto no alívio dos sintomas, quanto na promoção da independência e qualidade de vida. No entanto, protocolos sistematizados devem ser estabelecidos para a otimização do processo de reabilitação desses pacientes.

\section{Referências}

1. Fried LP, Tangen CM, Walston J, Newman AB, Hirsch C, Gottdiener J et al. Frailty in older adults: evidence for a phenotype. J Gerontol A Biol Scie Med S 2001;56(3):M146-56.

2. Woodhouse KW, Wynne H, Baillie S, James OF, Rawlins MD. Who are the frail elderly? O J Med 1988;68(255):505-6.

3. Winograd CH, Gerety MB, Chung M, Goldstein MK, Dominguez F Jr, Vallone $\mathrm{R}$ et al. Screening for frailty: criteria and predictors of outcomes. J Am Geriatr Soc 1991;39(8):778-84

4. Speechley M, Tinetti M. Falls and injuries in frail and vigorous community elderly persons. J Am Geriatr Soc 1991;39(1):46-52.
5. Campbell AJ, Buchner DM. Unstable disability and the fluctuations of frailty. Age Aging 1997;26(4):315-8

6. Strawbridge WJ, Shelma SJ, Balfour JL, Higby HR, Kaplan GA. Antecedents of frailty over three decades in an older cohort. J Gerontol B Psychol Sci Soc Sci 1998;53(1):9-16

7. Fried LP, Walston JM. Frailty and failure to thrive. In: Hazard WR, Blass JP, Ettinger WH Jr, Halter JB, Ouslander J (eds). Principles of Geriatric Medicine and Gerontology. $5^{\text {th }}$ ed. New York: McGraw-Hill; 2003.

8. Hamerman D. Toward an understanding of fraity. Ann Intern Med 1999;130(11):945-50. 
9. Rockwood K, Hogan DB, MacKnight C. Conceptualisation and measurement of frailty in elderly people. Drugs Aging 2000;17(4):295-302.

10. Lipsitz LA. Dynamics of stability: the physiologic basis of functional health and frailty. J Gerontol A Biol Sci Med 2002;57(3):B115-25.

11. Bortz WM. A conceptual framework of frailty. J Gerontol A Biol Sci Med Sci 2002;57(5):M283-8.

12. Espinoza $\mathrm{S}$, Walston JD. Frailty in older adults: insights and interventions. Cleve Clin J Med 2005;72(12):1105-12.

13. Morley JE, Haren MT, Rolland Y, Kim MJ. Frailty. Med Clin North Am 2006;90(5):837-47.

14. Ferrucci L, Guralnik JM, Studenski S, Fried LP, Cutler GB Jr, Walston JD et al. Designing randomized, controlled trials aimed at preventing or delaying functional decline and disability in frail, older persons: a consensus report. J Am Geriatr Soc 2004:52(4):625-34

15. Bergman H, Béland F, Karunananthan S, Hummel S, Hogan D, Wolfson C. Canadian iniciative on frailty and aging. Developing a working framework for understanding frailty. Gérontologie et Société 2004;109:15-29.

16. Teixeira INDO, Néri AL. A fragilidade no envelhecimento: fenômeno multidimensional, multideterminado e evolutivo. In: Freitas EV, Py L, Cançado F, Doll J, Gorzoni ML. Tratado de geriatria e geronlogia 2.ed. Rio de Janeiro: Guanabara Koogan: 2006, cap 115, p. 1102-9.

17. Fried LP, Hadley E, Walston JD, Newman AB, Guralnik JM, Studenski SA et al. From bedside to bench: research agenda for frailty. Sci Aging Knowledge Environ 2005;2005(31):pe24.

18. Fried LP, Walston W. Approach to the frail eldery patient. In: Kelley's Textbook of Internal Medicine. $4^{\text {th }}$ ed. Lippicontt Willians \& Wilkins; 2000.

19. Walston J, Hadley EC, Ferruci L, Guralnik JM, Newman AB, Studenski SA et al. Research agenda for frailty in older adults: toward a better understasnding of physiology an etiology: summary from the American Geriatrics Society/ National Institute on Aging Research Conference on Frailty in Olders Adults. J Am Geriatr Soc 2006;54(6):991-1001.

20. Silva TAA, Frisoli Jr A, Pinheiro MM, Szejnfeld VL. Sarcopenia and aging: aspects and therapeutic options. Rev Bras Reumatol 2006;46(6):391-7.

21. Roubenoff R. Catabolism of aging: is it inflammatory process? Curr Opin Clin Nutr Metab Care 2003:6(3):295-9

22. Brüünsgaard $H$, Pedersen BK. Age-related inflammatory cytokines and disease. Immunol Allergy Clin North Am 2003;23(1):15-39.

23. Leng SX, Cappola AR, Andersen RE, Blackman MR, Koening K, Blair M et al. Serum levels of insulin-like growth factor-1 (IGF-1) and dehydroepiandrosterone sulfate (DHEA-S), and their relationships with serum interleukin-6, in the geriatric syndrome of frailty. Aging Clin Exp Res 2004;16(2):153-7.

24. Harris TB, Ferruci L, Tracy RP, Corti MC, Wacholder S, Ettinger WH Jr et al. Associations of elevates interleukin-6 and C-reactive protein levels with mortality in the elderly. Am J Med 1999;106(5):506-12.

25. Roubenoff R, Parise H, Payette HA, Abad LW, D'Agostinho R, Jacques PF et al. Cytokines, insulin-like growth factor 1 , sarcopenia, and mortality in very old community-dwelling men and women: the Framingham Heart Study. Am J Med 2003;115(6):429-35.

26. Visser M, Pahor M, Taaffe DR, Goodpaster BH, Simonsick EM, Newman $A B$ et al. Relationship of interleukin- 6 and tumor necrosis factor-alpha with muscle mass and muscle strength in elderly men and women: the Health ABC Study. J Gerontol A Biol Sci Med Sci 2002;57(5): M326-32.

27. Walston J. Frailty: the search for underlying causes. Sci Aging Knowledge Environ 2004;2004(4):pe4.

28. Rawson ES, Wehnert ML, Clarkson PM. Effects of 30 days of creatine ingestion in older men. Eur J Appl Physiol Occup Physiol 1999;80(2):139-44.

29. Campbell WW, Joseph LJ, Davey SL, Cyr-Campbell D, Anderson RA, Evans WJ. Effects of resistence training and chromium picolinate on body composition and skeletal muscle in older men. J Appl Physiol 1999;86(1):29-39.

30. Daley MJ, Spinks WL. Exercise, mobility and aging. Sports Med 2000;29(1):1-12.

31. Fiatarone M, Marks EC, Ryan ND, Meredith CN, Lipsitz LA, Evans WJ. Highintensity strength training in nonagenarians. Effects on skeletal muscle. JAMA 1990;263(22):3029-34.

32. Okuma SS. 0 idoso e a atividade física: fundamentos e pesquisa. Campinas: Papirus; 1998.

33. Ades PA, Ballor DL, Ashikaga T, Utton JL, Nair KS. Weigth training improves walking endurance in healthy elderly persons. Ann Intern Med 1996;124(6):568-72.

34. Fiatarone MA, O'Neill EF, Ryan ND, Clements KM, Solares GR, Nelson ME et al. Exercise training and nutritional supplementation for physical frailty in very elderly people. N Engl J Med 1994;330(25):1769-775.

35. Hasten DL, Pak-Loduca J, Obert KA, Yarasheski KE. Resistance exercise acutely increases MHC and mixed muscle protein synthesis rates in 78-84 and 23-32 yr olds. Am J Physiol Endocrinol Metab 2000;278(4):E620-6.

36. Tribees S, Virtuoso JS. Prescription of physical exercises for eldery. Rev Saúde Com. 2005:1(12):163-72.

37. Borst SE. Interventions for sarcopenia and muscle weakness in older people. Age Ageing 2004;33(6):548-55

38. Buchner DM, Beresford SA, Larson EB, LaCroix AZ, Wagner EH. Effects of physical activity on health status in older adults. II. Intervention studies. Annu Rev Public Health 1992;13:469-88.

39. Lord SR, Sherrington C, Menz HB. Falls in older people: risk factors and strategies for prevention. United Kingdom: Cambridge University; 2001.

40. Sousa N, Marques U. Prevenção da queda do idoso. As alterações induzidas pelo treino da força no desempenho do Timed Get-Up \& Go Test e do Functional Reach Test. Revista Digital - Buenos Aires 2002;53(8). Disponível em: <http//www.efdesportes.com/efd53

41. Judge J0, Underwood M, Gennosa T. Exercise to improve gait velocity in older persons. Arch Phys Med Rehabil 1993;74(4):400-6. 